\title{
Marginal umbilical cord insertions in a bipartite placenta responsible for placenta abruption: a case report
}

\begin{abstract}
Hemorrhage is one of the major causes of maternal death. Main causes of APH are placenta previa, placenta abruption and uterine rupture. Rare causes of placenta abruption include marginal and velamentous umbilical cord insertions. We hereby present a case of placenta abruption due to marginal umbilical cord insertions occurring on a bipartite placenta. A 40-year-old nulliparous African woman, 35 weeks pregnant consulted for dark red pervaginal bleeding, which occurred recently. Past history was unremarkable. Her pregnancy was well followed up. A recent ultrasound scan revealed a fundal inserted placenta. Physical examination revealed a fundal height of $37 \mathrm{~cm}$, no uterine activity, normal fetal heart tones and a blood-stained vulva. Our diagnosis was a mild placenta abruption. An obstetrical ultrasound carried out revealed a normal pregnancy and a retroplacental blood clot of $11 \mathrm{~mm}$. A safe baby was born through an emergency cesarean section which revealed a normally inserted bipartite placenta with a $10 \%$ placenta detachment located on one placenta half and two cords inserted marginally. The postoperative period was uneventful and she was discharged five days after cesarean section. This case report shows that marginal cord insertion, which can lead to placenta abruption, can be also observed on a bipartite placenta.
\end{abstract}

Keywords: antepartum hemorrhage, marginal umbilical cord insertion, placenta abruption, placenta bipartite, cesarean section
Volume 12 Issue 5 - 202I

\author{
Elie Nkwabong,' Sylvie Borassi ${ }^{2}$ \\ IMD, Full Professor, Obstetrician \& Gynecologist, Department of \\ Obstetrics and Gynecology; Faculty of Medicine and Biomedical \\ Sciences \& University Teaching Hospital, Cameroon \\ ${ }^{2}$ Resident in Obstetrics \& Gynecology; Faculty of Medicine and \\ Biomedical Sciences, Cameroon
}

\begin{abstract}
Correspondence: Prof. Elie Nkwabong, MD, Full Professor, Obstetrician \& Gynecologist, Department of Obstetrics and Gynecology; Faculty of Medicine and Biomedical Sciences \& University Teaching Hospital, P.O. Box 1364 CHU Yaoundé, Cameroon, Tel (237) 699663843, Fax (237) 2223I2567, Email enkwabong@yahoo.fr
\end{abstract}

Received: September 21, 2021 | Published: October 19, 2021

\section{Introduction}

Bipartite placenta (BP) also called placenta bilobata, dimidiata, or duplex is a very rare anomaly in which, instead of one entire placenta, there are two placenta halves. ${ }^{1}$ Here, the cord usually divide itself into two, each one inserting on a placenta half, with frequently fetal membranes that unite the two placenta halves. Sometimes, there is just one umbilical cord that is inserted on one placenta half, which later gives blood vessel to the other placenta half through fetal membranes. As consequences, complications such as vasa praevia can occur, ${ }^{2}$ with risk of Benckiser's hemorrhage. ${ }^{3}$ The placenta halves are inserted at various distance from each other. They can also be inserted at various distances from the uterine lower segment.

The causes of BP are not well known. BP is frequently responsible for antepartum hemorrhage from a ruptured vasa praevia. Placenta abruption due to marginal umbilical cord insertion in a unique placenta is frequently observed. ${ }^{4}$ To the best of our knowledge, no case of placenta abruption on a BP has been reported. We hereby report a case of placenta abruption on a BP due to marginal umbilical cord insertions.

\section{Case presentation}

A 40-year-old African woman, G4P0030, 35 weeks gestation, consulted on $3^{\text {rd }}$ October 2019 for mild, dark-red first episode of pervaginal bleeding, of abrupt onset evolving for a few hours before consultation, without other associated symptoms. She had three missed abortions between 9 and 13 weeks' gestation of unknown causes, with manual vacuum aspiration (MVA) performed each time.
Past medical, gynecologic and surgical histories were unremarkable. She performed five antenatal consultations (ANC), the first being at 16 weeks of gestation. Antenatal biological work-ups were normal, but she did not receive any anti-anaemic prophylaxis. A standard ultrasound scan performed at 28 weeks diagnosed a normally inserted placenta.

Physical examination at admission revealed a healthy-looking patient, with a blood pressure of $135 / 80 \mathrm{mmHg}$, a pulse rate of 100/ min and a normal temperature. Conjunctiva were pink.

Abdominal examination revealed no uterine contractions on palpation. Fetal heart tones were regular at $138 \mathrm{bpm}$. The vulva was blood-stained. The rest of physical examination was unremarkable.

Our diagnosis was a probable mild placenta abruption. An emergency standard ultrasound revealed a retroplacental blood clot of $11 \mathrm{~mm}$. Her hemoglobin concentration was $11.3 \mathrm{~g} / \mathrm{dl}$. The Clotting profile was normal.

A live female baby with a birth weight of $2260 \mathrm{~g}$ and an Apgar score of 9 at the $5^{\text {th }}$ minute was delivered through an emergency cesarean section. The amniotic fluid was clear. The placenta was bipartite, one half located at the fundus and the other half containing a blood clot on the posterior uterine wall. Placenta examination at delivery showed that the umbilical cord divided itself into two, each part inserting on the margin of each placenta half (Figure 1). Further examination revealed a $10 \%$ placenta detachment on the half located on the posterior uterine wall. The postoperative course was without complications and she was discharged five days after cesarean section. 


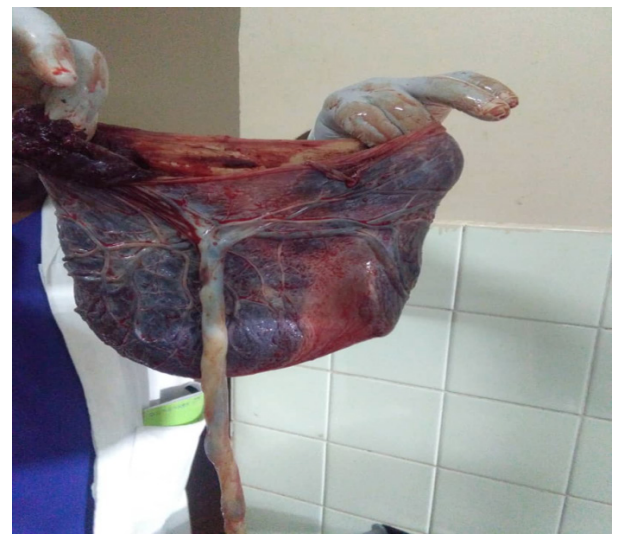

Figure I Two placenta halves joined by fetal membranes, the umbilical cord divides itself into two and each part insert on each placenta margin. The arrow shows the marginal retroplacental blood clot.

\section{Discussion}

Bipartite placenta is a placenta divided into two lobes of almost equal sizes. ${ }^{3}$ It is a rare placental anomaly without distinct racial, ethnic nor geographic predispositions. In bipartite placentas, there are frequently a velamentous or a marginal cord insertion (cord inserted $<2 \mathrm{~cm}$ from the placenta margin).

The pathogenesis of bipartite placenta is unclear. Due to the scarcity of articles in the literature, the risk factors for bipartite placenta are not well known. They include advanced maternal age ( $>35$ years), maternal history of infertility and In-Vitro Fertilization. ${ }^{5}$ Our patient was aged 40. There might be a link between MVA and BP, given that this procedure was done thrice in our patient. The pathogenesis of the bipartite placenta is unclear.

The antenatal diagnosis of bipartite placenta can be done by color Doppler obstetrical ultrasound. ${ }^{6,7}$ In our case, it was not diagnosed antenatally given that Doppler color technique was not done. Differential diagnosis is that of a succenturiate lobe, defined as one or more accessory placenta lobes distinct from the body of the placenta.

Bipartite placenta might be a cause of cesarean section if one placenta half covers the internal cervical os ${ }^{8,9}$ or if there is APH, as in our case where placenta abruption is explained by the presence of MCI. ${ }^{4}$ Postpartum hemorrhage is also possible if there is retention of one placenta half after childbirth.

\section{Conclusion}

BP is a rare morphological placental anormaly. This case report shows that placenta abruption can occur even on a BP. We recommend that women with APH should perform a Doppler color ultrasound scan, to enable the diagnosis of BP. Once diagnosed, such women should be closely monitored during pregnancy and labor.

\section{Acknowledgments}

None.

\section{Funding}

None.

\section{Conflicts of interest}

The authors did not report any potential conflicts of interest.

\section{References}

1. Ruiter L, Kok N, Limpens J, et al. Incidence of and risk indicators for vasa praevia:a systematic review. BJOG. 2016;123(8):1278-1287.

2. Quintero RA, Kontopoulos EV, Bornick PW, et al. In utero laser treatment of type II vasa previa. J Matern Fetal Neonatal Med. 2007;20(12):847851.

3. Régis C, Mubiayi ND, Devisme L, et al. Hémorragie de Benckiser:grave et inévitable?. J Gynecol Obstet Biol Reprod (Paris). 2006; $35(5 \mathrm{Pt}$ 1):517-521.

4. Nkwabong E, Njikam F, Kalla G. Outcome of pregnancies with marginal umbilical cord insertion. J Matern Fetal Neonatal Med. 2021;34(7):11331137

5. Isotton $\mathrm{AL}$, Salazar $\mathrm{CC}$, Peralta $\mathrm{CFA}$, et al. In vitro fertilization and vasa previa: a report of two cases. Rev Bras Ginecol Obstet. 2019;41(5):348351.

6. Stelzl PW, Yadav G, Perley L, et al. Early sonographic detection of a succenturiate placenta after IVF in a 42-year-old woman with multiple comorbidities. BMJ Case Rep. 2017;2017:bcr2017222189.

7. Cavaliere AF, Rosati P, Ciliberti P, et al. Succenturiate lobe of placenta with vessel anomaly:a case report of prenatal diagnosis and literature review. Clin Imaging. 2014;38(5):747-750.

8. Komatsu A, Kozuma S, Yoshida S, et al. A case of vasa previa diagnosed prenatally, and review of the literature. J Med Ultrason. 2011;38(1):4145 .

9. Shukunami K, Tsunezawa W, Hosokawa K, et al. Placenta previa of a succenturiate lobe:a report of two cases. Eur J Obstet Gynecol Reprod Biol. 2001;99(2):276-277. 